International Journal of Engineering \& Technology, $7(4.10)(2018) 1046-1049$
International Journal of Engineering \& Technology
SPC
Website: www.sciencepubco.com/index.php/IJET
Research paper

\title{
Kamenev-Type Oscillation Criteria for Generalized Second Order Sublinear $\alpha$-Difference Equations
}

\author{
A.Benevatho Jaison ${ }^{1 *}$, Sk.Khadar Babu², V.Chandrasekar ${ }^{3}$ \\ ${ }^{1,2}$ Vellore Institute of Technology, Vellore - 632 014, Tamil Nadu, India \\ ${ }^{3}$ Thiruvalluvar University College of Arts and Science, Thennangur - 604 408, Tamil Nadu, India \\ *Corresponding author E-mail:benejaison@gmail.com
}

\begin{abstract}
By means of Riccati transformation techniques, authors establish some new oscillation criteria for generalized second order nonlinear $\alpha$-difference equation $\Delta_{\alpha(\ell)}\left(p(k \ell+j)\left(\Delta_{\alpha(\ell)}(u(k \ell+j))\right)^{\gamma}\right)+q(k \ell+j) u^{\beta}((k-\sigma) \ell+j)=0$, when $0<\beta<1$ and $\gamma$ are quotient of odd positive integers.
\end{abstract}

Keywords: Delay; Sublinear; Superlinear.

\section{Introduction}

Difference equations represent a fascinating mathematical area on its own as well as a rich field of the applications in such diverse disciplines. For general background as difference equations with many examples from diverse fields, one can refer to [1].

The theory of difference equations is based on the operator $\Delta$ defined as $\Delta u(k)=u(k+1)-u(k), k \in \mathrm{N}=\{0,1,2, \cdots\}$. Even though some authors [1] have suggested the definition of $\Delta$ as

$$
\Delta u(k)=u(k+\ell)-u(k), \quad \ell \in(0, \infty)
$$

no significant progress took place on this line. Jerzy Popenda, et.al., [16] defined $\Delta_{\alpha}$ as $\Delta_{\alpha} u(k)=u(k+1)-\alpha u(k)$. Authors in [14], considering the operator $\Delta$ defined by (E) as $\Delta_{\ell}$ many interesting results in number theory were obtained [14]. In [15], they generalized the definition of $\Delta_{\alpha}$ by $\Delta_{\alpha(\ell)}$ defined as $\Delta_{\alpha(\ell)} u(k)=u(k+\ell)-\alpha u(k)$ for the real valued function $u(k)$ and $\ell \in(0, \infty)$ and also obtained the solutions of certain types of generalized $\alpha$-difference equations.

In recent years, the asymptotic behaviour of second order difference equations has been the subject of investigations by many authors [2-12,17-19,21-22].

In this paper, we will be concerned with a class of generalized second order sublinear delay difference equations of the form

$\Delta_{\alpha(\ell)}\left(p(k \ell+j)\left(\Delta_{\alpha(\ell)}(u(k \ell+j))\right)^{\gamma}\right)$

$+q(k \ell+j) u^{\beta}((k-\sigma) \ell+j)=0$,

where $\Delta_{\alpha(\ell)}$ denotes the forward difference operator for any real valued function $u(k \ell+j), \quad k \in(0, \infty), \quad \ell \in(0, \infty)$, $j=k-\left[\frac{k}{\ell}\right] \ell, \quad \gamma \quad$ is quotient of odd positive integers, $0<\beta<1$ is quotient of odd positive integers, $\sigma$ is a fixed nonnegative integer, $p(k \ell+j)>0$, and $q(k \ell+j) \geq 0$ are real valued functions, and for some $k_{0}>0$,

$\sum_{k=k_{0}}^{\infty}\left(\frac{1}{p(k \ell+j)}\right)^{1 / \gamma}=\infty$,

By a solution of (1) we mean a nontrivial real valued function $u(k)$ defined for $k \geq-\sigma$, and satisfies equation (1) for $k \in(0, \infty)$. Clearly, if

$u(k \ell+j)=A(k \ell+j)$ for $k \in[-\sigma, 0]$

are given, then equation (1) has a unique solution satisfying the initial condition (3).

\section{Main Results}

Theorem 1 Assume that (2) holds. Furthermore, assume that there exist a positive real valued functions $\rho(k \ell+j)$ such that for every $\eta \geq 1$ and positive number $M$.

$$
\begin{aligned}
& \limsup _{k \rightarrow \infty} \sum_{r=0}^{k}\left[\rho(r \ell+j) q(r \ell+j)-\alpha(p((r-\sigma) \ell+j)){ }^{1 / \gamma}\right. \\
& \left.\times \frac{\eta^{1-\beta}((r-\sigma+1) \ell+j)^{1-\beta}\left(\Delta_{\ell} \rho(r \ell+j)\right)^{2}}{4 \beta(M)^{(\gamma-1) / \gamma} \rho(r \ell+j)}\right]
\end{aligned}
$$

Then every solution of equation (1) oscillates.

Proof. Suppose to the contrary that $u(k \ell+j)$ is an eventually nonoscillatory solution of (1) such that $u((k-\sigma) \ell+j)>0$ for 
all $k \geq k_{0}>0$. We shall consider only this case, since the substitution $v(k \ell+j)=-u(k \ell+j)$ transforms equation (1) into an equation of the same form. From equation (1) we have for $k \geq k_{0}$

$$
\begin{aligned}
& \Delta_{\alpha(\ell)}\left(p(k \ell+j)\left(\Delta_{\alpha(\ell)} u(k \ell+j)\right)^{\gamma}\right) \\
& =-q(k \ell+j) u^{\beta}((k-\sigma) \ell+j) \leq 0,
\end{aligned}
$$

and so $p(k \ell+j)\left(\Delta_{\alpha(\ell)} u(k \ell+j)\right)^{\gamma}$ is an eventually nonincreasing sequence. We first show that $p(k \ell+j)\left(\Delta_{\alpha(\ell)} u(k \ell+j)\right)^{\gamma} \geq 0$ for $k \geq k_{0}$. In fact, if there exists an real $k_{1} \geq k_{0}$ such that $p\left(k_{1} \ell+j\right)\left(\Delta_{\alpha(\ell)} u\left(k_{1} \ell+j\right)\right)^{\gamma}=c<0$, then (5) implies that $p(k \ell+j)\left(\Delta_{\alpha(\ell)} u(k \ell+j)\right)^{\gamma} \leq c \quad$ for $\quad k \geq k_{1}$ that is $\Delta_{\alpha(\ell)} u(k \ell+j) \leq(c / p(k \ell+j))^{1 / \gamma}$ hence as $k \rightarrow \infty$ $u(k \ell+j) \leq \alpha u\left(k_{1} \ell+j\right)$

$+\sum_{r=k_{1}}^{k-1}\left[(\alpha-1) u(r \ell+j)+\left(\frac{1}{p(r \ell+j)}\right)^{1 / \gamma}\right] \rightarrow-\infty$

which contradicts the fact that $u(k \ell+j)>0$ for $k \geq k_{0}$, then $p(k \ell+j)\left(\Delta_{\alpha(\ell)} u(k \ell+j)\right)^{\gamma} \geq 0$. Also we claim that $\Delta_{\alpha(\ell)}^{2} u(k \ell+j) \leq 0$. If not there exists $k_{1} \geq k_{0}$ such that $\Delta_{\alpha(\ell)}^{2} u(k \ell+j)>0$ for $k \geq k_{1}$ and this implies that $\Delta_{\alpha(\ell)} u((k+1) \ell+j)>\Delta_{\alpha(\ell)} u(k \ell+j), \quad$ so that since $\Delta_{\alpha(\ell)} p(k \ell+j) \geq 0, \quad p((k+1) \ell+j)\left(\Delta_{\alpha(\ell)} u((k+1) \ell+j)\right)^{\gamma}$ $>p((k+1) \ell+j)\left(\Delta_{\alpha(\ell)} u(k \ell+j)\right)^{\gamma}$

$\geq p(k \ell+j)\left(\Delta_{\alpha(\ell)} u(k \ell+j)\right)^{\gamma}$ and this contradicts the fact that $p(k \ell+j)\left(\Delta_{\alpha(\ell)} u(k \ell+j)\right)^{\gamma}$ is nonincreasing sequence, then $\Delta_{\alpha(\ell)}^{2} u(k \ell+j) \leq 0$, and therefore we have for $k \geq k_{0}$

$$
u(k \ell+j)>0, \Delta_{\alpha(\ell)} u(k \ell+j) \text { and } \Delta_{\alpha(\ell)}^{2} u(k \ell+j) \leq 0 .
$$

Define the sequence $z(k \ell+j)$ by

$$
z(k \ell+j)=\alpha^{\left[\frac{k}{\ell}\right]-1} \frac{\rho(k \ell+j) p(k \ell+j)\left(\Delta_{\alpha(\ell)} u(k \ell+j)\right)^{\gamma}}{u^{\beta}((k-\sigma) \ell+j)}
$$

then $z(k \ell+j)>0$, and

$$
\begin{aligned}
& \Delta_{\alpha(\ell)} z(k \ell+j)=\frac{\alpha^{\left[\frac{k}{\ell}\right]} \rho(k \ell+j) \Delta_{\ell}\left(p(k \ell+j)\left(\Delta_{\alpha(\ell)} u(k \ell+j)\right)^{\gamma}\right)}{u^{\beta}((k-\sigma) \ell+j)} \\
& +p((k+1) \ell+j)\left(\Delta_{\alpha(\ell)} u((k+1) \ell+j)\right) \Delta_{\alpha(\ell)}\left(\frac{\alpha^{\left[\frac{k}{\ell}\right]-1} \rho(k \ell+j)}{u^{\beta}((k-\sigma) \ell+j)}\right)(9)
\end{aligned}
$$

From (1) and (9), we have
$\Delta_{\ell} z(k \ell+j)=-\rho(k \ell+j) q(k \ell+j)+\frac{\Delta_{\ell} \rho(k \ell+j) z((k+1) \ell+j)}{\rho((k+1) \ell+j)}$

$-\frac{\alpha^{\left[\frac{k}{\ell}\right]} \rho(k \ell+j) p((k+1) \ell+j)\left(\Delta_{\alpha(\ell)} u((k+1) \ell+j)\right)^{\gamma}}{u^{\beta}((k-\sigma+1) \ell+j)}$

$\times \frac{\Delta_{\ell} u^{\beta}((k-\sigma) \ell+j)}{u^{\beta}((k-\sigma) \ell+j)}$

From (5) and (7), we get

$p((k-\sigma) \ell+j)\left(\Delta_{\ell} u((k-\sigma) \ell+j)\right)^{\gamma}$

$\geq p((k+1) \ell+j)\left(\Delta_{\ell} u((k+1) \ell+j)\right)^{\gamma}$

and $u((k+1-\sigma) \ell+j) \geq u((k-\sigma) \ell+j)$

and then from (10) and (11), we have

$\Delta_{\alpha(\ell)} z(k \ell+j) \leq-\rho(k \ell+j) q(k \ell+j)$

$+\frac{\Delta_{\ell} \rho(k \ell+j) z((k+1) \ell+j)}{\rho((k+1) \ell+j)}$

$-\frac{\rho(k \ell+j) p((k+1) \ell+j)\left(\Delta_{\alpha(\ell)} u((k+1) \ell+j)\right)^{\gamma}}{\left(u^{\beta}((k-\sigma+1) \ell+j)\right)^{2}}$

$\times \Delta_{\alpha(\ell)} \alpha^{\left[\frac{k}{\ell}\right]-1} u^{\beta}((k-\sigma) \ell+j)$

Now, by using the inequality in [2], for all $u \neq v>0$ and for $0<\beta \leq 1, \quad u^{\beta}-v^{\beta} \geq \beta u^{\beta-1}(u-v)$. Then, we have

$\Delta_{\alpha(\ell)} \alpha^{\left[\frac{k}{\ell}\right]-1} u^{\beta}((k-\sigma) \ell+j)$

$=\beta(u((k-\sigma+1) \ell+j))^{\beta-1} \Delta_{\alpha(\ell)} \alpha^{\left[\frac{k}{\ell}\right]-1} u((k-\sigma) \ell+j)$

Substitute from (13) in (12), we have

$\Delta_{\alpha(\ell)} z(k \ell+j) \leq-\rho(k \ell+j) q(k \ell+j)$

$+\frac{\Delta_{\ell} \rho(k \ell+j) z((k+1) \ell+j)}{\rho((k+1) \ell+j)}$

$-\left(\frac{\rho(k \ell+j) p((k+1) \ell+j) \beta(u((k+1-\sigma) \ell+j))^{\beta-1}}{\left(u^{\beta}((k-\sigma+1) \ell+j)\right)^{2}}\right)$

$\times \Delta_{\alpha(\ell)} u((k-\sigma) \ell+j)\left(\Delta_{\alpha(\ell)} u((k+1) \ell+j)\right)^{\gamma}$

From (11) and (14), we have

$\Delta_{\alpha(\ell)} z(k \ell+j) \leq-\rho(k \ell+j) q(k \ell+j)$

$+\frac{\Delta_{\ell} \rho(k \ell+j) z((k+1) \ell+j)}{\rho((k+1) \ell+j)}$

$-\frac{\beta \rho(k \ell+j)(p((k+1) \ell+j))^{1 / \gamma} p((k+1) \ell+j)}{(p((k-\sigma) \ell+j))^{1 / \gamma}(u((k-\sigma+1) \ell+j))^{1-\beta}}$

$\times \frac{\left(\Delta_{\alpha(\ell)} u((k+1) \ell+j)\right)^{\gamma+1}}{\left(u^{\beta}((k-\sigma+1) \ell+j)\right)}$

$\Delta_{\alpha(\ell)} z(k \ell+j) \leq-\rho(k \ell+j) q(k \ell+j)$

$+\frac{\Delta_{\ell} \rho(k \ell+j) z((k+1) \ell+j)}{\rho((k+1) \ell+j)}$

$-\left(\frac{\beta \rho(k \ell+j)(p((k+1) \ell+j))^{(1 / \gamma)-1}(p((k+1) \ell+j))^{2}}{(p((k+1) \ell+j))^{2}(p((k-\sigma) \ell+j))^{1 / \gamma}(u((k-\sigma+1) \ell+j))^{1-\beta}}\right.$ 


$$
\left.\times \frac{(\rho((k+1) \ell+j))^{2}\left(\Delta_{\alpha(\ell)} u((k+1) \ell+j)\right)^{2 \gamma}}{\left(u^{\beta}((k-\sigma+1) \ell+j)\right)^{2}\left(\Delta_{\alpha(\ell)} u((k+1) \ell+j)\right)^{\gamma-1}}\right)
$$

From (7), we conclude that $u(k \ell+j) \leq \alpha u\left(k_{0} \ell+j\right)+\Delta_{\alpha(\ell)} u\left(k_{0} \ell+j\right)\left(\left(k-k_{0}\right) \ell+j\right), k \geq k_{0}$ and consequently there exists a $k_{1} \geq k_{0}$ and appropriate constant $\eta \geq 1$ such that $u(k \ell+j) \leq \eta(k \ell+j)$ for $k \geq k_{1}$ and this implies that $u((k-\sigma v+1 v) \ell v+j) \leq \eta((k-\sigma+1) \ell+j)$ for $k \geq k_{2}=k_{1}+\sigma-1$ and, hence

$$
\frac{1}{(u((k-\sigma+1) \ell+j))^{1-\beta}} \geq \frac{1}{(\eta((k-\sigma+1) \ell+j))^{1-\beta}} .
$$

Since $p(k \ell+j)\left(\Delta_{\alpha(\ell)} u(k \ell+j)\right)^{\gamma}$ is a positive and increasing function, there exists a $k_{2} \geq k_{1}$ sufficiently large such that $p(k \ell+j)\left(\Delta_{\alpha(\ell)} u(k \ell+j)\right)^{\gamma} \leq \frac{1}{M}$ for some positive constant $M \quad$ and $k \geq k_{2}$, and hence by (5) we have $p((k+1) \ell+j)\left(\Delta_{\alpha(\ell)} u((k+1) \ell+j)\right)^{\gamma} \leq \frac{1}{M}$, so that

$\frac{1}{\left(\Delta_{\alpha(\ell)} u((k+1) \ell+j)\right)^{\gamma-1}} \geq(M p((k+1) \ell+j))^{(\gamma-1) / \gamma}$

Then from (8), (15), (16) and (17) we have

$$
\begin{aligned}
& \Delta_{\alpha(\ell)} z(k \ell+j) \leq-\rho(k \ell+j) q(k \ell+j) \\
& +\frac{\Delta_{\ell} \rho(k \ell+j) z((k+1) \ell+j)}{\rho((k+1) \ell+j)} \\
& -\frac{\beta \rho(k \ell+j) M^{(\gamma-1) / \gamma}(z((k+1) \ell+j))^{2}}{(p((k+1) \ell+j))^{2}(p((k-\sigma) \ell+j))^{1 / \gamma} \eta^{1-\beta}((k-\sigma+1) \ell+j)^{1-\beta}}
\end{aligned}
$$

$\Delta_{\alpha(\ell)} z(k \ell+j) \leq-\rho(k \ell+j) q(k \ell+j)+$

$$
+\frac{(p((k-\sigma) \ell+j))^{1 / \gamma} \eta^{1-\beta}((k-\sigma+1) \ell+j)^{1-\beta}\left(\Delta_{\ell} \rho(k \ell+j)\right)^{2}}{4 \beta(M)^{(\gamma-1) / \gamma} \rho(k \ell+j)}
$$$$
-\left[\frac{\sqrt{\beta(M)^{(\gamma-1) / \gamma} \rho(k \ell+j)} z((k+1) \ell+j)}{\rho((k+1) \ell+j) \sqrt{\eta((k-\sigma+1) \ell+j)^{1-\beta} p((k-\sigma) \ell+j)}}\right.
$$$$
\left.\frac{\sqrt{\eta^{1-\beta}((k-\sigma+1) \ell+j)^{1-\beta}(p((k-\sigma) \ell+j))^{1 / \gamma}} \Delta_{\ell} \rho(k \ell+j)}{2 \sqrt{\beta(M)^{(\gamma-1) / \gamma} \rho(k \ell+j)}}\right]^{2}
$$

$<-[\rho(k \ell+j) q(k \ell+j)$

$$
\left.-\frac{(p((k-\sigma) \ell+j))^{1 / \gamma} \eta^{1-\beta}((k-\sigma+1) \ell+j)^{1-\beta}\left(\Delta_{\ell} \rho(k \ell+j)\right)^{2}}{4 \beta(M)^{(\gamma-1) / \gamma} \rho(k \ell+j)}\right]
$$

$\sum_{r=0}^{k}[\rho(r \ell+j) q(r \ell+j)$

$$
\left.-\frac{(p((r-\sigma) \ell+j))^{1 / \gamma} \eta^{1-\beta}((r-\sigma+1) \ell+j)^{1-\beta}\left(\Delta_{\ell} \rho(r \ell+j)\right)^{2}}{4 \beta(M)^{(\gamma-1) / \gamma} \rho(r \ell+j)}\right]<c_{1}
$$

for all large $k$, and this is contrary to (4). The proof is complete.

Theorem 2 Assume that (2) holds. Let $\rho(k \ell+j)$ be a real valued function. Furthermore, we assume that there exists a double function $H(m, k \ell+j) ; m \geq k \geq 0$ such that $(i) H(m, m)=0$ for $m \geq 0$, (ii) $H(m, k \ell+j)>0$ for $m>k \ell+j \geq 0$, (iii) $\Delta_{2 \alpha(\ell)} H(m, k \ell+j)=H(m,(k+1) \ell+j)-\alpha H(m, k \ell+j) \leq 0$ for $m \geq k \ell+j \geq 0$. If

$$
\limsup _{m \rightarrow \infty} \frac{1}{H(m, 0)} \sum_{k=k_{0}}^{m-1}[H(m, k \ell+j) \rho(k \ell+j) q(k \ell+j)
$$$$
-\frac{(\rho((k+1) \ell+j))^{2}}{\overline{\rho(k \ell+j)}}
$$

$\left.\times\left(h(m, k \ell+j)-\frac{\Delta_{\ell} \rho(k \ell+j)}{\rho((k+1) \ell+j)} \sqrt{H(m, k \ell+j)}\right)^{2}\right]=\infty$,

where $h(m, k \ell+j)=-\frac{\Delta_{2(\ell)} H(m, k \ell+j)}{\sqrt{H(m, k \ell+j)}}$,

$$
\overline{\rho(k \ell+j)}=\frac{\beta \rho(k \ell+j) M^{(\gamma-1) / \gamma}}{(p((k-\sigma) \ell+j))^{1 / \gamma} \eta^{1-\beta}((k-\sigma+1) \ell+j)^{1-\beta}}
$$

Then every solution of equation (1) oscillates.

Proof. We proceed as in Theorem 1, we assume that equation (1) has a nonoscillatory solution, say $u((k-\sigma) \ell+j)>0$ for all $k \geq k_{0}$. From (18) we have for $k \geq k_{2}$,

$\rho(k \ell+j) q(k \ell+j) \leq-\Delta_{\alpha(\ell)} z(k \ell+j)$

$+\frac{\Delta_{\ell} \rho(k \ell+j) z((k+1) \ell+j)}{\rho((k+1) \ell+j)}-\frac{\overline{\rho(k \ell+j)}}{(\rho((k+1) \ell+j))^{2}} w^{2}(k+\ell)$.

$\sum_{k=n}^{m-1} H(m, k \ell+j) \rho(k \ell+j) q(k \ell+j) \leq H(m, k \ell+j) z(k \ell+j)$

$+\sum_{k=n}^{m-1} z((k+1) \ell+j) \Delta_{2(\ell)} H(m, k \ell+j)$

$+\sum_{k=n}^{m-1} H(m, k \ell+j) \frac{\Delta_{\ell} \rho(k \ell+j) z((k+1) \ell+j)}{\rho((k+1) \ell+j)}$

$-\sum_{k=n}^{m-1} \frac{H(m, k \ell+j) \overline{\rho(k \ell+j)} z^{2}((k+1) \ell+j)}{(\rho((k+1) \ell+j))^{2}}$

$=H(m, k \ell+j) z(k \ell+j)-\sum_{k=n}^{m-1}[z((k+1) \ell+j)$

$+\frac{\rho((k+1) \ell+j)}{2 \sqrt{H(m, k \ell+j) \overline{\rho(k \ell+j)}}}$

$\left.\times\left(h(m, k \ell+j) \sqrt{H(m, k \ell+j)}-\frac{\Delta_{\ell} \rho(k \ell+j) H(m, k \ell+j)}{\rho((k+1) \ell+j)}\right)\right]^{2}$

$+\frac{1}{4} \sum_{k=n}^{m-1} \frac{(\rho((k+1) \ell+j))^{2}}{\overline{\rho(k \ell+j)}}$

$\times\left(h(m, k \ell+j)-\frac{\Delta_{\ell} \rho(k \ell+j) \sqrt{H(m, k \ell+j)}}{\rho((k+1) \ell+j)}\right)^{2}$.

$\limsup _{m \rightarrow \infty} \frac{1}{H(m, 0)} \sum_{k=0}^{m-1}[H(m, k \ell+j) \rho(k \ell+j) q(k \ell+j)$

$-\frac{(\rho((k+1) \ell+j))^{2}}{\rho(k \ell+j)}$

$\left.\times\left(h(m, k \ell+j)-\frac{\Delta_{\ell} \rho(k \ell+j) \sqrt{H(m, k \ell+j)}}{\rho((k+1) \ell+j)}\right)^{2}\right]<0$,

which contradicts to our assumption. Hence the proof.

\section{References}

[1] Agarwal RP, Difference Equations and Inequalities, Marcel Dekker, New York (2000). 
[2] Benevatho Jaison A, Khadar Babu Sk (2016), Oscillation for generalized first order nonlinear difference equations. Global Journal of Pure and Applied Mathematics 12(1), 51-54.

[3] Benevatho Jaison A, Khadar Babu Sk (2016), Kamenev-type oscillation criteria for second order generalized delay difference equations. International Journal of Control Theory and Applications 9(28), 463-469.

[4] Benevatho Jaison A, Khadar Babu Sk (2016), Oscillation for generalized first order nonlinear a-difference equations. International Journal of Pure and Applied Mathematics 109(7), 67-74.

[5] Benevatho Jaison A, Khadar Babu Sk (2017), Oscillation theorems for generalized second-order nonlinear delay difference equations. International Journal of Pure and Applied Mathematics 113(9), 8492.

[6] Benevatho Jaison A, Khadar Babu Sk (2017), Oscillation theorems for generalized second kind nonlinear delay difference equations. International Journal of Pure and Applied Mathematics 115(9), 25 36.

[7] Benevatho Jaison A, Khadar Babu Sk (2017), Oscillatory behavior of generalized nonlinear difference equations. Global Journal of Pure and Applied Mathematics 13(1), 205-209.

[8] Benevatho Jaison A, Khadar Babu Sk (2017), Oscillatory behavior of generalized Nonlinear difference equations. Global Journal of Pure and Applied Mathematics 13(2), 415-423.

[9] Benevatho Jaison A, Khadar Babu Sk (2018), Kamenev-type oscillation criteria for generalized sublinear delay difference equations. International Journal of Pure and Applied Mathematics 118(10) 135-145.

[10] Benevatho Jaison A, Khadar Babu Sk (2018), Oscillation for generalized second kind nonlinear delay a-difference equations. International Journal of Pure and Applied Mathematics 118(23), 507-515.

[11] Chandrasekar V, Srimanju V (2016), Oscillation for generalized second order sublinear neutral delay alpha difference equations. Global Journal of Pure and Applied Mathematics 12(1), 55-59.

[12] Chandrasekar V, Srimanju V (2016), Qualitative properties of discrete version of generalized kneser's and arzela-ascoli's theorems. International Journal of Control Theory and Applications 9(28), 549-554.

[13] Hardy GH, Littlewood JE, Polya G, Inequalities, 2nd edn., Cambridge University Press, Cambridge (1952).

[14] Maria Susai Manuel M, Britto Antony Xavier G, Thandapani E (2006), Theory of generalized difference operator and its applications. Far East Journal of Mathematical Science 20(2), 163171.

[15] Maria Susai Manuel M, Chandrasekar V, Britto Antony Xavier G (2011), Solutions and applications of certain class of $\alpha$-difference equations. International Journal of Applied Mathematics 4(6), 943 954.

[16] Popenda J, Szmanda B (1984), On the oscillation of solutions of certain difference equations. Demonstratio Mathematica 14(1), 153-164.

[17] Srimanju V, Khadar Babu Sk (2017), Oscillatory criteria for generalized second-order quasilinear neutral delay difference equations. International Journal of Pure and Applied Mathematics 113(9), 7583.

[18] Srimanju V, Khadar Babu Sk (2017), Oscillation of generalized quasilinear difference equations. International Journal of Pure and Applied Mathematics 115(9), 37-45.

[19] Srimanju V, Khadar Babu Sk (2017), Oscillation criteria for generalized quasi-linear difference equations. Global Journal of Pure and Applied Mathematics 13(1), 210-216.

[20] Srimanju V, Khadar Babu Sk (2017), Oscillation criteria for generalized second kind quasi-linear neutral a-difference equations. Global Journal of Pure and Applied Mathematics 13(2), 544-551.

[21] Srimanju V, Khadar Babu Sk (2018), Oscillatory properties of third-order quasilinear generalized difference equations. International Journal of Pure and Applied Mathematics 118(10), 155-165.

[22] Srimanju V, Khadar Babu Sk (2018), Oscillation of generalized quasilinear a-difference equations. International Journal of Pure and Applied Mathematics 118(23), 497-505. 